\title{
The dependence of plasma density in the topside ionosphere on the solar activity level
}

\author{
L. Liu, W. Wan, X. Yue, B. Zhao, B. Ning, and M.-L. Zhang \\ Institute of Geology and Geophysics, Chinese Academy of Sciences, Beijing 100029, China \\ Received: 28 November 2006 - Revised: 31 March 2007 - Accepted: 28 May 2007 - Published: 29 June 2007
}

\begin{abstract}
In this paper, the ten-year (1996-2005) total ion density $N_{i}$ measurements from the Defense Meteorological Satellite Program (DMSP) spacecraft in the morning and evening (09:30 and 21:30 LT) sectors have been analyzed to explore the dependence of plasma densities in the topside ionosphere at middle and low latitudes on the solar activity level. Results indicate that there is a strong solar activity dependence of DMSP $N_{i}$ at $848 \mathrm{~km}$ altitude, which has latitudinal and seasonal features. The plasma density in the topside ionosphere has an approximately linear dependence on daily F107 and a strongly nonlinear dependence on SEM/SOHO EUV, such that the change rate of $N_{i}$ becomes greater with increasing solar EUV. This is quite different from the dependence of $N_{i}$ near the F-Region peak $(N m \mathrm{~F} 2)$, at which the rate of change of $N m \mathrm{~F} 2$ decreases with increasing solar EUV. The rate of change of $N_{i}$ at the DMSP altitude is greatest in the latitude range where $N_{i}$ is greatest during high solar activity. We suggest that this greater rate of change (or amplification effect) of $N_{i}$ at the DMSP altitude is mainly a consequence of the solar activity variations of the topside scale height. The changes in the height of the F-Region peak $\left(h m \mathrm{~F}_{2}\right)$ and the density $N m \mathrm{~F} 2$ play a secondary role.
\end{abstract}

Keywords. Solar physics, astrophysics, and astronomy (U1traviolet emissions) - Ionosphere (Plasma temperature and density; Solar radiation and cosmic ray effects)

\section{Introduction}

It is well known that the temporal variations of the Earth's ionosphere are ultimately linked to those of solar activity, because the main source of the ionospheric plasma is photoionization of neutrals by solar extreme ultraviolet (EUV) and X-ray radiations. Previous studies have shown that electron density in the ionosphere varies with solar activity in a

Correspondence to: L. Liu

(liul@mail.iggcas.ac.cn) rather complicated way (e.g. Balan et al., 1996; Kane, 2003; Liu et al., 2003; Richards, 2001; Sethi et al., 2002; Su et al., 1999; West et al., 1997). Considerable progress has been achieved in understanding the solar cycle variations of the $\mathrm{F}$ layer (e.g. Kane, 2003; Lei et al., 2005; Liu et al., 2006b; Sethi et al., 2002), while limited analyses have been applied to the topside ionosphere (e.g. Rich et al., 2003; Su et al., 1999; West et al., 1997; Zhao et al., 2005). Evidence indicates that ionospheric variations have altitude dependencies (Rich et al., 2003; Su et al., 1999). For example, Su et al. (1999) found strong altitude dependencies in the solar activity variations of electron densities, which were observed with the Japanese incoherent scatter radar. Recently, Rich et al. (2003) revealed that the 27-day effect is much more pronounced in the topside plasma density than that in the total electron content (TEC).

Topside plasma densities have been being continuously measured by the Defense Meteorological Satellite Program (DMSP) spacecraft since 1987. This database is ideally suited for studies of the climatology of the topside ionosphere. At the same time, since 1996, the solar EUV fluxes in 26-34 $\mathrm{nm}$ and $0.1-50 \mathrm{~nm}$ wavelength bands have been being continuously monitored by the Solar EUV Monitor (SEM) spectrometer on board the Solar Heliospheric Observatory (SOHO) (Judge et al., 1998). There are few comparable records in history, especially the solar EUV observations (Kane, 2003). In this analysis, data from both measurements are collected to investigate the solar cycle variations of the topside ionosphere. We will focus on the effects in the morning and evening (09:30 and 21:30 LT) sectors at middle and low latitudes. The most striking new feature is that DMSP $N_{i}$ increases at a higher rate with increasing SEM/SOHO EUV. This is contrary to the saturation effects of the peak electron density of the F layer $(N m \mathrm{~F} 2)$ and TEC (e.g. Balan et al., 1994, 1996; Liu et al., 2003, 2006b). This feature is pronounced in a broad range of latitudes, which has not been reported yet. 


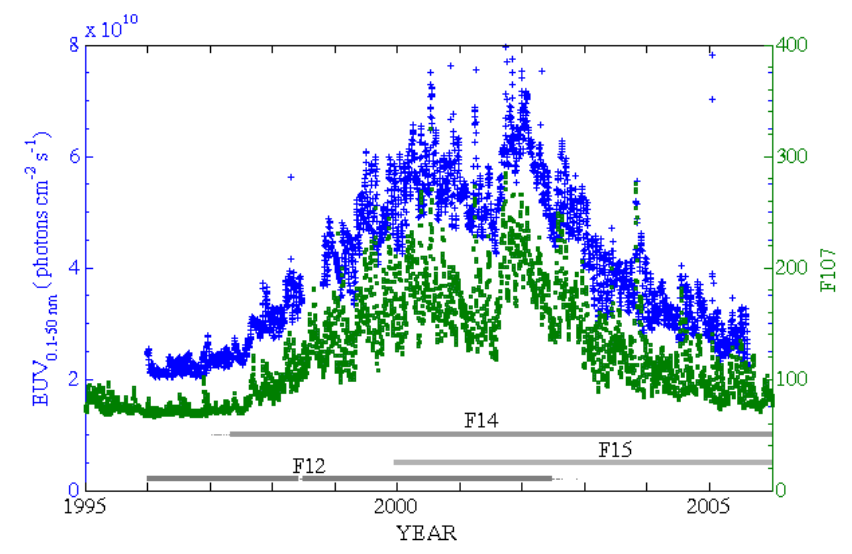

Fig. 1. Periods of DMSP $N_{i}$, and SEM/SOHO EUV in the 0.1$50 \mathrm{~nm}$ wavelength band with corresponding solar index F107.

\section{Data source}

The series of the DMSP spacecraft are designated with letter $\mathrm{F}$ and the flight number. The fleet of DMSP spacecraft are in Sun-synchronous polar orbits at a constant altitude of about $848 \mathrm{~km}$. The period of an orbit is around $101 \mathrm{~min}$, and consecutive orbits are separated in longitude by $25.5^{\circ}$. The nearly constant local time of the DMSP orbital planes makes their ionospheric measurements unique for allowing other drivers of the plasma characteristics to be more noticeable. The overlapped operational time of the spacecraft ensures the data's integrity. The spacecraft carries a "Special Sensor-Ions, Electrons and Scintillation" (SSIES) package to monitor the behavior of thermal plasma in the topside ionosphere since 1987. This package has been described in many works (e.g. Rich et al., 2003; West et al., 1997). The sum of plasma densities over all species (referred to as the total ion density, or $N_{i}$ ) is measured with the onboard Scintillation Meter at a resolution of $24 \mathrm{~Hz}$.

In this paper, only data in the morning and evening (09:30 and 21:30 LT) sectors from the spacecraft F12, F14, and F15 were chosen. The $N_{i}$ data are archived and provided at the University of Texas, Dallas (UTD) website as a 4-s average. At each local time sector, values of $N_{i}$ in each day from 1996 to 2005 between $\pm 50^{\circ}$ geomagnetic latitude are averaged in 51 latitude bins. The geomagnetic coordinates we used are provided by UTD. The magnetic latitudes are the corrected geomagnetic coordinates of the sub-spacecraft locations. Each bin includes data points over all longitudes, is centered at even latitudes $\left(50^{\circ} \mathrm{N}, 48^{\circ} \mathrm{N}, \ldots, 48^{\circ} \mathrm{S}, 50^{\circ} \mathrm{S}\right)$, and spans $2.5^{\circ}$ in extent (some overlap with neighbor bins). Irregularity structures, e.g. equatorial plasma density bubbles, may appear in the post-sunset topside ionosphere. Irregularities in the data set have been removed before taking the average. The seasons are defined as December solstice ( \pm 45 days centered on 21 December), March equinox $( \pm 30$ days centered on 23 March), June solstice ( \pm 45 days cen- tered on 21 June), and September equinox ( \pm 30 days centered on 23 September). Moreover, Zhao et al. (2005) examined the storm effects for more than one hundred cases during 1996-2004 and found that $\mathrm{Ni}$ is enhanced during the main phases of storms and depressed during the recovery phases of storms. As a matter of fact, since the topside ionosphere is largely controlled by solar EUV, the geomagnetic effect is less statistically evident. It is found that there is no obvious statistical relationship between $N_{i}$ and geomagnetic disturbances. Therefore, we ignore the geomagnetic activity effects on the plasma densities in this analysis.

Since 1996, solar EUV fluxes in 26-34-nm and 0.1-50-nm wavelength bands have been being continuously monitored by SEM/SOHO (Judge et al., 1998). The daily values of the SEM/SOHO EUV fluxes are available at the website http: //www.usc.edu/dept/space_science/semdatafolder/long/. We only use the 0.1-50-nm EUV flux data (denoted as $\boldsymbol{I}$ in the following sections for brevity) in this study, since there is a strong linear cross-correlation between these two bands (Liu et al., 2006b). The 10.7-cm solar radio flux, F107, is often used as a standard proxy for solar activity. In this paper, we adopt the adjusted values of F107 provided at the SPIDR website in accord with the work of Liu et al. (2006b), since different F107 values (the observed, adjusted and absolute values) do not affect our conclusions. Figure 1 shows the variations of F107 and the intensities of EUV in the 0.1-50$\mathrm{nm}$ wavelength band. The time coverage of DMSP $N_{i}$ is also displayed in Fig. 1.

\section{Results}

As an example, Fig. 2 shows scatter plots of DMSP $N_{i}$ against $\boldsymbol{I}$, and F107 in every day during December solstices at 5 magnetic latitude bins $\left(40^{\circ} \mathrm{S}, 16^{\circ} \mathrm{S}, 0^{\circ}, 16^{\circ} \mathrm{N}\right.$, and $\left.40^{\circ} \mathrm{N}\right)$. Due to the page limitation, we only show data in the morning sector here, although this feature presents at both local time sectors (21:30 and 09:30 LT). Readers may consult the general differences between both local time sectors in the work of Zhao et al. (2005). A complete picture over the latitudinal coverage is illustrated in Fig. 3. The solid curves in Fig. 2 show the corresponding fits with the piecewise linear leastsquares method. To obtain the nonlinear trend, we choose 11 pieces of fitted bits in each latitudinal bin in this analysis. Although the detailed response in each latitude bin is slightly different, the overall results show that $N_{i}$ increases with increasing F107 or $\boldsymbol{I}$. Plasma densities increase almost linearly with F107 when F107 lies in the range 100 to 250. The variation of $N_{i}$ at an altitude of $848 \mathrm{~km}$ with F107 is generally similar to that at $600 \mathrm{~km}$ (Su et al., 1999). The different manifestations of $N_{i}$ with F107 and $\boldsymbol{I}$ may be understood when a nonlinear statistical relationship between F107 and $\boldsymbol{I}$ is taken into account. Results illustrated in Fig. 2 of Liu et al. (2006b) show that the increase in SEM/SOHO EUV with 

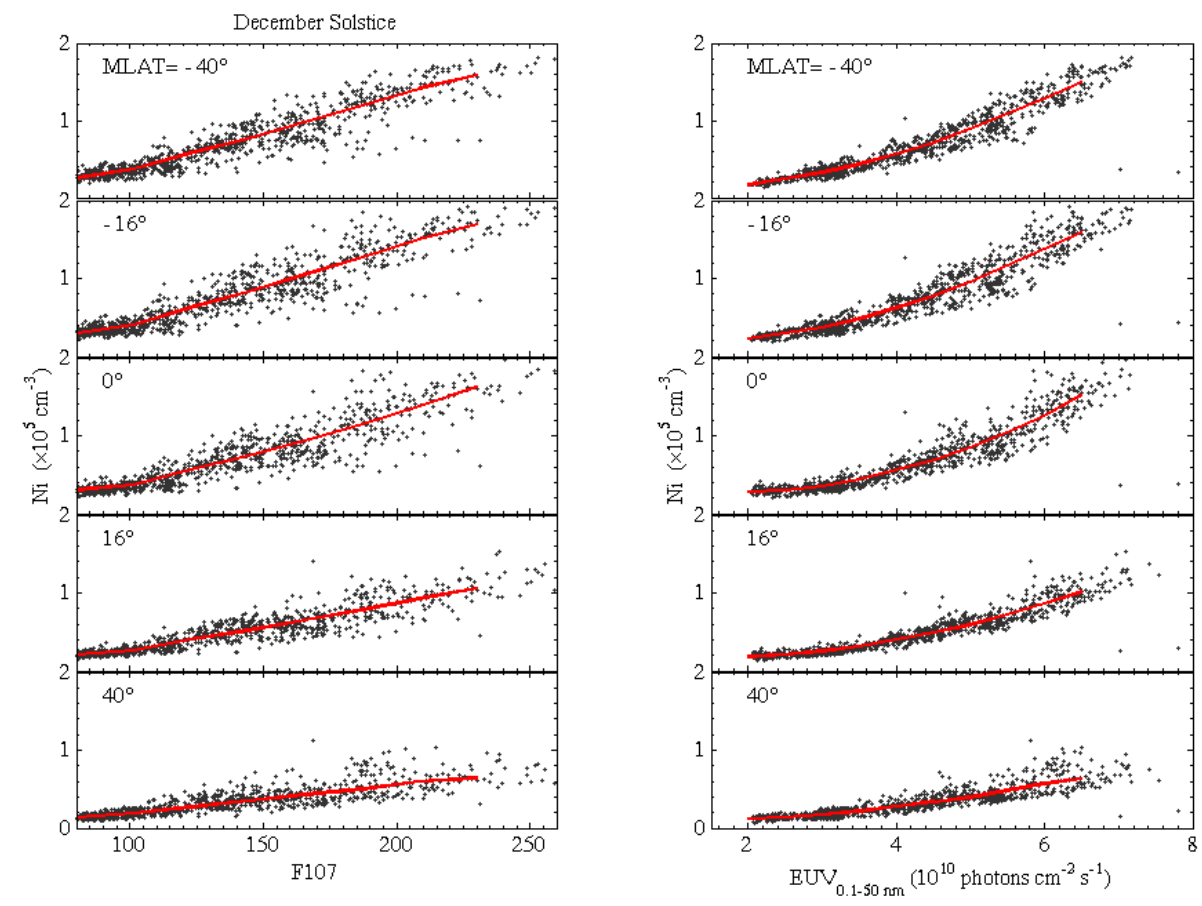

Fig. 2. Variations of the morning (09:30 LT) DMSP $N_{i}$ with F107 and SEM/SOHO EUV during December solstices. The variations of piecewise least-squares fit are shown by the solid curves.
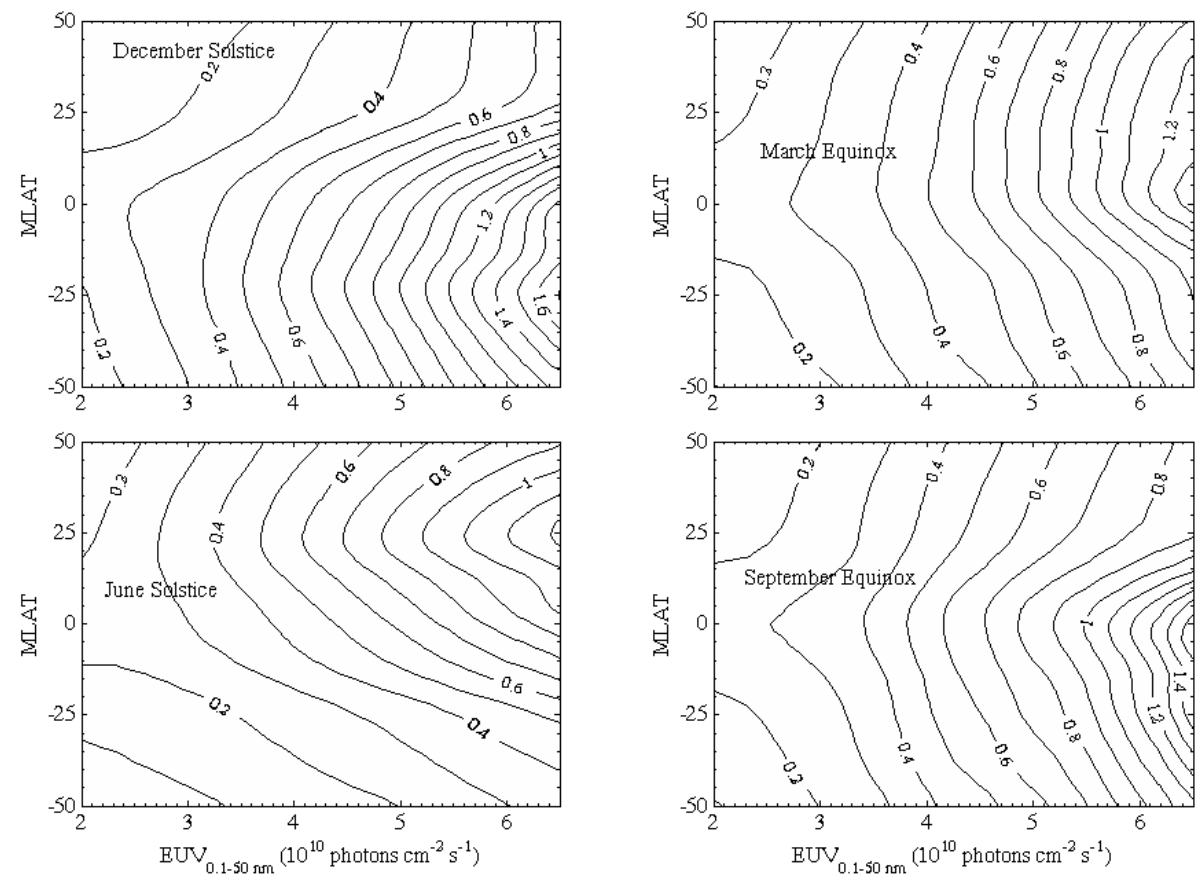

Fig. 3. The piecewise linear least-square fitted values of DMSP $N_{i}\left(\right.$ in $10^{5} \mathrm{~cm}^{-3}$ ) as functions of magnetic latitude and solar EUV in four seasons.

F107 is much steeper at low and moderate F107 values than at high ones.

What is important to be noticed here is contrary to the saturation effect in the $\mathrm{F}$ region (that is, plasma densities near the F peak tend to increase much less or even negatively for higher F107 or EUV) (e.g. Balan et al., 1996; Liu et al., 2003, 2006b), DMSP $N_{i}$ increases at a higher rate with increasing EUV. This feature of a greater rate of change (we call it 

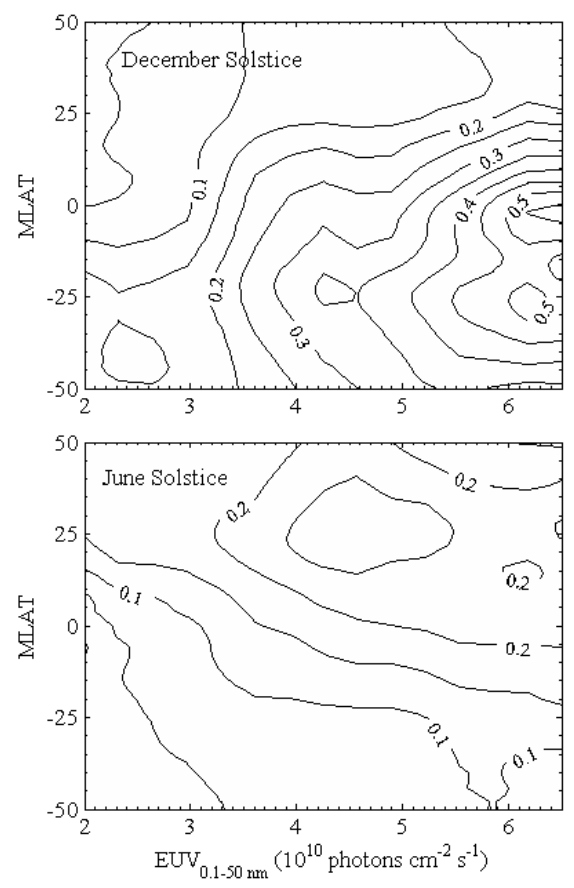
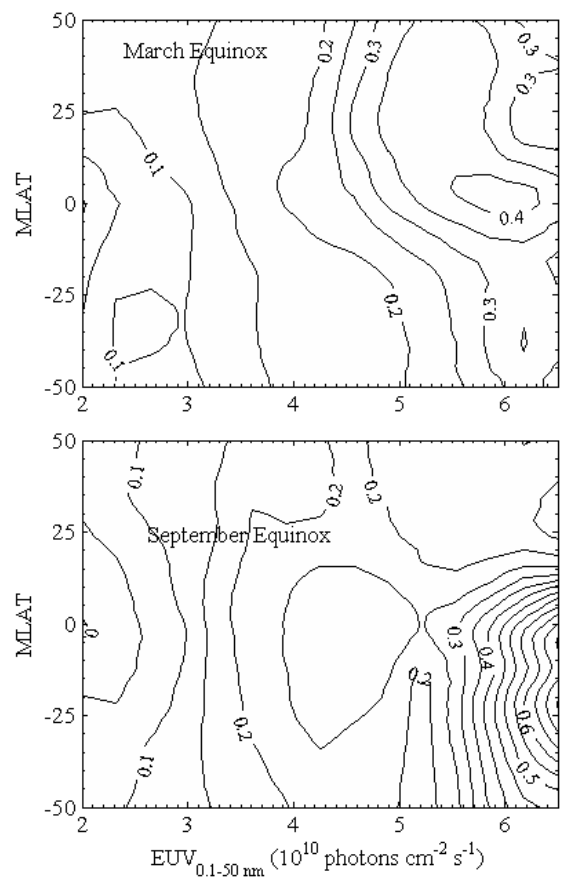

Fig. 4. The change rates of $N_{i}$ with respect to solar EUV, $\mathrm{d} N_{i} / \mathrm{d} \boldsymbol{I}$ (in $10^{5} \mathrm{~cm}^{-3}$ per $10^{10}$ photons cm $\mathrm{cm}^{-1}$ ), as functions of magnetic latitude and solar EUV in four seasons.

an amplification effect) is more significant in the Southern Hemisphere and at lower magnetic latitude in the December solstice months. This noticeable feature also exists in other seasons (see Figs. 3 and 4).

Figure 3 illustrates the latitudinal variations and solar activity dependences of the piecewise fitted $N_{i}$ in the four seasons in the morning sector. Adjusting the size of the fitting pieces does not change the general feature, while these tests indicate some influences on the corresponding change rates of $N_{i}$ with $\boldsymbol{I}, \mathrm{d} N_{i} / \mathrm{d} \boldsymbol{I}$. It is clearly seen in Fig. 3 that DMSP $N_{i}$ has distinct seasonal and latitudinal variations and strong solar activity dependencies.

The plasma densities at $848-\mathrm{km}$ altitude have highest values within a broad range of latitudes around the magnetic equator, which shifts with season. At higher latitudes, plasma densities are found to decrease with increasing latitude in both hemispheres and plasma density is higher in the summer hemisphere.

The corresponding change rates of $N_{i}$ with $\boldsymbol{I}, \mathrm{d} N_{i} / \mathrm{d} \boldsymbol{I}$ (in unit of $10^{5} \mathrm{~cm}^{-3}$ per $10^{10}$ photons $\mathrm{cm}^{-2} \mathrm{~s}^{-1}$ ), in the four seasons are plotted in Fig. 4. As illustrated in Fig. 4, d $N_{i} / \mathrm{d} \boldsymbol{I}$ varies with latitude and season. Moreover, the values of $\mathrm{d} N_{i} / \mathrm{d} \boldsymbol{I}$ tend to increase with increasing solar EUV. For example, at $20^{\circ} \mathrm{S}$ magnetic latitude in the September equinox the value of $\mathrm{d} N_{i} / \mathrm{d} \boldsymbol{I}$ increases from lower than $10^{4} \mathrm{~cm}^{-3}$ per $10^{10}$ photons $\mathrm{cm}^{-2} \mathrm{~s}^{-1}$ to higher than $7 \times 10^{4} \mathrm{~cm}^{-3}$ per $10^{10}$ photons $\mathrm{cm}^{-2} \mathrm{~s}^{-1}$. In other words, the increase of $N_{i}$ becomes stronger at high EUV values than at low values. This feature is more distinct in December solstice (the top-left panel of Fig. 4) and September equinox (the bottom-right panel of Fig. 4).

Figures 3 and 4 show that the maximum ion density in the Southern Hemisphere during the December solstices is larger than that in the Northern Hemisphere during the June solstices. The hemispheric asymmetry becomes stronger with increasing solar activity. The latitudinal features of the DMSP plasma densities are obviously modulated by solar activity and season, that is, the latitudinal structure becomes more significant at higher solar activity and at seasons other than equinoxes. As we know, the latitudinal changes in the solar zenith angle (SZA) could have some effects on the latitudinal pattern shown in Figs. 3 and 4. The local sunrise time could change by $2 \mathrm{~h}$ from $50^{\circ} \mathrm{N}$ to the geographic equator; over the same latitude range SZA could change up to $45^{\circ}$ in winter. Moreover, the movement of the ionosphere due to neutral winds may also be a primary cause of the latitudinal and seasonal variations of the topside plasma density (Venkatraman and Heelis, 2000). Equinoctial asymmetries seen in the MU radar observations (Balan et al., 1998) are also found in DMSP $N_{i}$. Readers can consult the work of Liu et al. (2007b) on the hemispheric and annual asymmetries of DMSP Ni. Furthermore, a statistical model of DMSP $N_{i}$ has been developed by Zhao et al. (2005) using an empirical orthogonal function analysis. 


\section{Discussion}

The topside ionosphere at DMSP altitude is mainly composed of ions $\mathrm{O}^{+}, \mathrm{H}^{+}$, and $\mathrm{He}^{+}$. The abundances of these ions are dominated by transport and chemical processes (e.g. Chandra and Rangaswamy, 1967; West et al., 1997; Zhao et al., 2005). The plasma distribution of the equatorial and midlatitude ionosphere is subject to a number of transport processes involving thermospheric neutral winds, $\boldsymbol{E} \times \boldsymbol{B}$ drifts, and field-aligned diffusions (Venkatraman and Heelis, 2000). During the daytime, photoionization of $\mathrm{O}^{+}$in the F-region creates an upward pressure gradient force in the topside ionosphere. As a result, the newly-created $\mathrm{O}^{+}$diffuses upward along the magnetic field lines. The plasma also undergoes an upward $\boldsymbol{E} \times \boldsymbol{B}$ drift motion during the daytime. The upward diffusion and $\boldsymbol{E} \times \boldsymbol{B}$ drift of plasma during the daytime cause the $\mathrm{O}^{+}$ion to become the dominant species at the DMSP altitudes, especially at high solar activity (West et al., 1997). At night, rapid recombination of the ion species in the ionosphere decreases the upward diffusion of the topside plasma along the field line. Above the F-layer peak, the transport processes become more and more dominant in controlling the plasma distribution. As a result, it is expected that, to first approximation, plasma density $\left(N_{e}\right)$ in the topside ionosphere is under diffusive equilibrium (Rishbeth and Garriott, 1969):

$$
\begin{aligned}
N_{e}(h) & =N m \mathrm{~F} 2 \exp \left[0.5\left(1-z-e^{-z}\right)\right], \\
z & =(h-h m \mathrm{~F} 2) / H(h) .
\end{aligned}
$$

Here $h m \mathrm{~F} 2$ is the peak height and $H(h)$ the topside plasma scale height at an altitude $h$. Thus, variations of plasma densities in the topside ionosphere are closely coupled with those in the F-region, as well as with the topside scale height, although the behavior in the topside ionosphere is rather different from that in the F-region.

Based on Eq. (1), a qualitative explanation for different manifestations of the solar activity variations in the topside and the F-region ionosphere is made by taking into account the variations of (1) $N m \mathrm{~F} 2$, (2) $h m \mathrm{~F} 2$, and (3) topside scale height with increasing solar activity.

When solar activity becomes more active, the photoionization production rate increases due to the enhancement of both solar EUV flux and the concentration of atomic oxygen (e.g. Ivanov-Kholodny and Mikhailov, 1986; Richards, 2001). This will contribute to the increase of $\mathrm{O}^{+}$and total ion concentration $N_{i}$ (or electron density) at a fixed altitude in the ionosphere or around $h m \mathrm{~F} 2$, although the saturation effect may occur or becomes significant at some locations (Balan et al., 1996; Liu et al., 2003, 2006b; Richards, 2001; Su et al., 1999).

At the same time, the height of the $F$ peak is closely related with solar activity, that is, the $F_{2}$ peak also moves to higher altitude at higher solar activity (e.g. Buonsanto, 1990; Lei et al., 2005; Liu et al., 2006b; Pandey et al., 2003; Zhang et al., 2005). Higher $h m \mathrm{~F} 2$ will lead to a smaller reduced

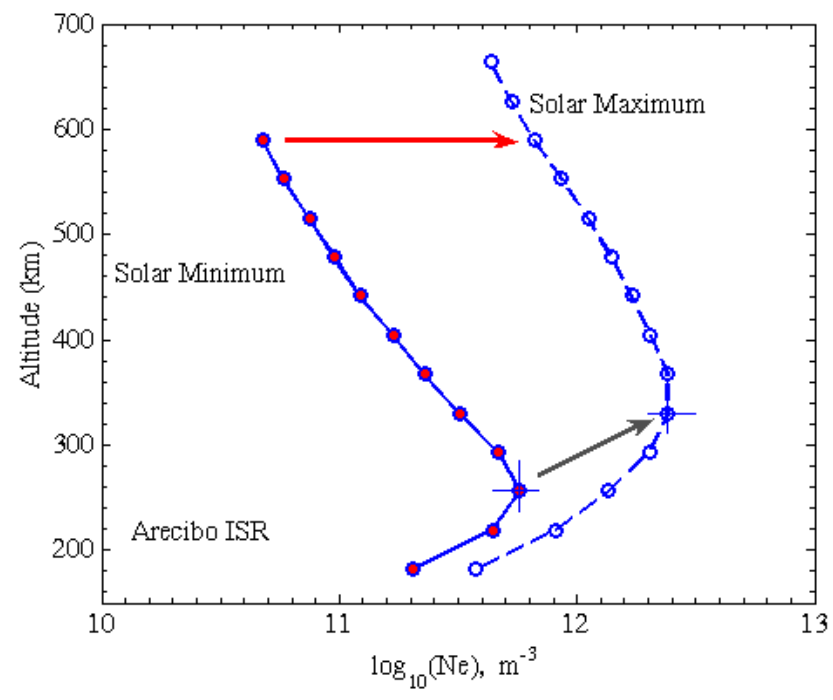

Fig. 5. Typical electron density profiles over Arecibo under solar maximum (April 1989) and solar minimum conditions (April 1986).

height $z$ in Eq. (1), thus also giving rise to plasma density at the DMSP altitude.

Moreover, the shape of the topside plasma density profiles or the scale height may also vary with solar activity (Chuo, 2007; Kutiev et al., 2006; Kutiev and Marinov, 2007; Liu et al., 2007a; Luan et al., 2006; Stankov and Jakowski, 2006). With measurements from incoherent scatter radar, digisonde, topside sounder, and radio occultation techniques, the plasma scale heights around the $F$ peak and the topside ionosphere are found to become larger at higher solar activity (Kutiev et al., 2006; Lei et al., 2005; Liu et al., 2006a, 2007a; Stankov and Jakowski, 2006), which will also increase plasma density at the DMSP altitude as a key role by further decreasing the reduced height $z$ in Eq. (1).

The aforementioned three related variations are schematically illustrated in Fig. 5 with typical electron density profiles observed with the Arecibo incoherent scatter radar under solar minimum and maximum conditions. A complete statistical analysis on the scale heights from the Arecibo ISR observations is reported by Liu et al. (2007a). Since DMSP $N_{i}$ observations are made at an altitude of several scale heights above the $F_{2}$ peak, variations in density near the $F_{2}$ peak may be amplified in DMSP plasma density through the "pivot effect" (Rich et al., 2003), e.g. the 27-day variation. The "pivot effect" occurs when a variation in density is amplified by a factor of $e(=2.71828)$ at an altitude of a scale height above the $F_{2}$ peak. Because DMSP observations come from more than 3 scale heights above the $F_{2}$ peak, Rich et al. suggested that this effect is contributed to by scale height. As a result, even in a situation where the solar activity dependency of the electron density near the $F_{2}$ peak is weak, with increasing altitude, the variation of plasma density will be amplified and a stronger solar activity dependency will be present at 
the DMSP altitude. On the basis of Eq. (1), the amplification comes from the exponential term, which is mainly contributed from the solar activity variations of scale height. As illustrated in Fig. 5, the contributions from the larger scale heights, as well as higher $\mathrm{F}$ layer and enhanced ionization in the F-region causes the amplification effect of the topside $N_{i}$ at a fixed altitude.

On the other hand, the amplification feature of DMSP $N_{i}$ is not due to altitude changes because the mean of the altitude of the DMSP spacecraft remains almost constant. The altitude of the DMSP spacecraft decreases by approximately $0.25 \mathrm{~km} /$ year during solar minimum and approximately $0.50 \mathrm{~km} /$ year at solar maximum. Over a ten-year span the altitude change would be insignificant compared to a scale height of more than $100 \mathrm{~km}$.

\section{Summary}

In this paper the dependence of plasma densities in the topside ionosphere on solar activity level has been investigated by analyzing the DMSP total ion density, the SEM/SOHO EUV, and solar proxy F107 from 1996 to 2005. The present investigation reveals that DMSP $N_{i}$ at $848 \mathrm{~km}$ is rather sensitive to solar EUV flux, which has latitudinal and seasonal features. The most noticeable feature is that the topside plasma density is approximately linear against F107, but it is nonlinear with the intensities of the SEM/SOHO EUV. The change rate of $N_{i}$ with increasing solar EUV is more pronounced around the magnetic latitudes where maximum values of $N_{i}$ occur at high solar activity. We suggest that this amplification effect of the solar activity variations of $N_{i}$ in the topside ionosphere mainly results from solar activity variations of the topside scale heights. The changes in the height and electron density of the underlying ionosphere with solar activity are also part of the reason, but not the primary reason. The combined effects result in the amplification feature of $N_{i}$ in the topside ionosphere. However, a quantitative determination of the source mechanisms requires further investigation with theoretical models which will be done later.

Acknowledgements. The authors thank two referees providing valuable comments to improve the presentation of the manuscript. The DMSP data are provided by the Center for Space Sciences at University of Texas at Dallas and the US Air Force. The SEM/SOHO data is provided by Space Sciences Center of University of Southern California. The F107 index is taken from the SPIDR web site. The Arecibo incoherent scatter radar data are provided by the Arecibo Observatory, which is operated by the Cornell University under an agreement with the National Science Foundation. This research was supported by National Natural Science Foundation of China (40674090, 40636032), the KIP Pilot Project (kzcx3-sw-144) of Chinese Academy of Sciences, and National Important Basic Research Project (2006CB806306).

Topical Editor M. Pinnock thanks S. Zhang and another anonymous referee for their help in evaluating this paper.

\section{References}

Balan, N., Bailey, G. J., Jenkins, B., Rao, P. B., and Moffett, R. J.: Variations of ionospheric ionization and related solar fluxes during an intense solar cycle, J. Geophys. Res., 99(A2), 22432253, 1994.

Balan, N., Bailey, G. J., and Su, Y. Z.: Variations of the ionosphere and related solar fluxes during solar cycles 21 and 22, Adv. Space Res., 18(3), 11-14, 1996.

Balan, N., Otsuka, Y., Bailey, G. J., and Fukao, S.: Equinoctial asymmetries in the ionosphere and thermosphere observed by the MU radar, J. Geophys. Res., 103(A5), 9481-9495, 1998.

Buonsanto, M. J.: Observed and calculated F2 peak heights and derived meridional winds at mid-latitudes over a full solar cycle, J. Atmos. Terr. Phys., 52, 223-240, 1990.

Chandra, S. and Rangaswamy, S.: Geomagnetic and solar control of ionization at $1000 \mathrm{~km}$, J. Atmos. Terr. Phys., 29, 259-265, 1967.

Chuo, Y. J.: The variation of ionospheric slab thickness over equatorial ionization area crest region, J. Atmos. Solar-Terr. Phys., 69, 947-954, 2007.

Ivanov-Kholodny, G. S. and Mikhailov, A. V.: The prediction of ionospheric conditions, D. Reidel Publishing Company, Holland, 1986.

Judge, D., McMullin, D. R., Ogawa, H. S., Hovestadt, D., Klecker, B., Hilchenbach, M., Mobius, E., Canfield, L. R., Vest, R. E., Watts, R., Tarrio, C., Kuhne, M., and Wurz, P.: First Solar EUV Irradiances Obtained from SOHO by the SEM, Solar Phys., 177, 161-173, 1998.

Kane, R. P.: Solar EUV and ionospheric parameters: A brief assessment, Adv. Space Res., 32(9), 1713-1718, 2003.

Kutiev, I. and Marinov, P.: Topside sounder model ofscale height and transition height characteristics of the ionosphere, Adv. Space Res., 39(5), 759-766, 2007.

Kutiev, I. S., Marinov, P. G., and Watanabe, S.: Model of topside ionosphere scale height based on topside sounder data, Adv. Space Res., 37, 943-950, 2006.

Lei, J., Liu, L., Wan, W., and Zhang, S.-R.: Variations of electron density based on long-term incoherent scatter radar and ionosonde measurements over Millstone Hill, Radio Sci., 40, RS2008, doi:10.1029/2004RS003106, 2005.

Liu, J. Y., Chen, Y. I., and Lin, J. S.: Statistical investigation of the saturation effect in the ionospheric foF2 versus sunspot, solar radio noise, and solar EUV radiation, J. Geophys. Res., 108(A2), 1067, doi:10.1029/2001JA007543, 2003.

Liu, L., Le, H., Wan, W., Sulzer, M. P., Lei, J., and Zhang, M.-L.: An analysis of the scale heights in the lower topside ionosphere based on the Arecibo incoherent scatter radar measurements, J. Geophys. Res., 112, A06307, doi:10.1029/2007JA012250, 2007a.

Liu, L., Zhao, B., Wan, W., Venkartraman, S., Zhang, M.-L., and Yue, X.: Yearly variations of global plasma densities in the topside ionosphere at middle and low latitudes, J. Geophys. Res., 112, doi:10.1029/2007JA012283, in press, 2007b.

Liu, L., Wan, W., and Ning, B.: A study of the ionogram derived effective scale height around the ionospheric $h m \mathrm{~F} 2$, Ann. Geophys., 24, 851-860, 2006a.

Liu, L., Wan, W., Ning, B., Pirog, O. M., and Kurkin, V. I.: Solar activity variations of the ionospheric peak electron density, J. Geophy. Res., 111, A08304, doi:10.1029/2006JA011598, 2006b.

Luan, X., Liu, L., Wan, W., Lei, J., Zhang, S-R, Holt, J. M., and 
Sulzer, M. P.: A study of the shape of the topside electron density profile derived from incoherent scatter radar measurements over Arecibo and Millstone Hill, Radio Sci., 41, RS4006, doi:10.1029/2005RS003367, 2006.

Pandey, V. K., Sethi, N. K., and Mahajan, K. K.: Dependence of F2- peak height on solar activity: A study with incoherent scatter measurements, Adv. Space Res., 31(3), 543-548, 2003.

Rich, F. J., Sultan, P. J., and Burke, W. J.: The 27-day variations of the plasma densities and temperatures in the topside ionosphere, J. Geophys. Res., 108(A7), 1297, doi:10.1029/2002JA009731, 2003.

Richards, P. G.: Seasonal and solar cycle variations of the ionospheric peak electron density: comparison of measurement and models, J. Geophys. Res., 106(A12), 12 803-12 819, 2001.

Rishbeth, H. and Garriott, O. K.: Introduction to ionospheric physics, 331 pp., Academic Press, New York, 1969.

Sethi, N. K., Goel, M. K., and Mahajan, K. K.: Solar cycle variations of foF2 from IGY to 1990, Ann. Geophys., 20, 1677-1685, 2002, http://www.ann-geophys.net/20/1677/2002/.

Stankov, S. M. and Jakowski, N.: Topside ionospheric scale height analysis and modeling based on radio occultation measurements, J. Atmos. Solar-Terr. Phys., 68, 134-162, 2006.
Su, Y. Z., Bailey, G. J., and Fukao, S.: Altitude dependencies in the solar activity variations of the ionospheric electron density, J. Geophys. Res., 104(A7), 14 879-14 891, 1999.

Venkatraman, S. and Heelis, R.: Interhemispheric plasma flows in the equatorial topside ionosphere, J. Geophys. Res., 105(A8), 18 457-18 464, 2000.

West, K. H., Heelis, R. A., and Rich, F. J.: Solar activity variations in the composition of the low-latitude topside ionosphere, J. Geophys. Res., 102(A1), 295-305, 1997.

Zhang, S.-R., Holt, J. M., van Eyken, A. P., McCready, M., AmoryMazaudier, C., Fukao, S., and Sulzer, M.: Ionospheric local model and climatology from long-term databases of multiple incoherent scatter radars, Geophys. Res. Lett., 32, L20102, doi:10.1029/2005GL023603, 2005.

Zhao, B., Wan, W., Liu, L., Yue, X., and Venkatramn, S.: Statistical characteristics of the total ion density in the topside ionosphere during the period 1996-2004 using empirical orthogonal function (EOF) analysis, Ann. Geophys., 23, 3615-3631, 2005, http://www.ann-geophys.net/23/3615/2005/. 